\title{
Octet baryon mass splittings from up-down quark mass differences
}

\author{
R. Horsley ${ }^{* a}$, J. Najjar ${ }^{b}$, Y. Nakamura ${ }^{c}$, D. Pleiter ${ }^{d b}$, P. E. L. Rakow ${ }^{e}$, G. Schierholz ${ }^{f}$ \\ and J. M. Zanotti ${ }^{g}$ \\ a School of Physics and Astronomy, University of Edinburgh, Edinburgh EH9 3JZ, UK \\ ${ }^{b}$ Institut für Theoretische Physik, Universität Regensburg, 93040 Regensburg, Germany \\ ${ }^{c}$ RIKEN Advanced Institute for Computational Science, Kobe, Hyogo 650-0047, Japan \\ d JSC, Jülich Research Centre, 52425 Jülich, Germany \\ ${ }^{e}$ Theoretical Physics Division, Department of Mathematical Sciences, University of Liverpool, \\ Liverpool L69 3BX, UK \\ ${ }^{f}$ Deutsches Elektronen-Synchrotron DESY, 22603 Hamburg, Germany \\ ${ }^{g}$ CSSM, School of Chemistry and Physics, University of Adelaide, Adelaide SA 5005, Australia \\ E-mail: rhorsley@ph.ed.ac.uk
}

\section{QCDSF-UKQCD Collaboration}

\begin{abstract}
Using an SU(3) flavour symmetry breaking expansion in the quark mass, we determine the QCD component of the neutron-proton, Sigma and Xi mass splittings of the baryon octet due to updown (and strange) quark mass differences. Provided the average quark mass is kept constant, the expansion coefficients in our procedure can be determined from computationally cheaper simulations with mass degenerate sea quarks and partially quenched valence quarks. Full details and numerical results are given in [1].
\end{abstract}

The 30th International Symposium on Lattice Field Theory - Lattice 2012,

June 24-29, 2012

Cairns, Australia

* Speaker. 


\section{Introduction}

Isospin symmetry was introduced by Heisenberg in the 1930s to explain non-electromagnetic similarities between the proton and neutron. Nowadays, of course, this is ascribed to the $u$ and $d$ quarks having similar mass and the same strong - or QCD - interactions. This $S U(2)$ flavour symmetry is not exact, there are isospin breaking effects, due to

- the $m_{d}-m_{u}$ quark mass difference which is a 'pure' QCD effect,

- a QED component due to the different quark charges.

As both effects are small then we can set

$$
M^{\exp }=M^{*}+M^{Q E D},
$$

where we denote by a * the 'pure' QCD component. There is an interplay between effects: electromagnetic (EM) effects tend to make $p$ heavier than $n$, but $m_{d}-m_{u}$ works in the opposite direction and in fact dominates as the neutron is heavier than the proton, $\left(M_{n}-M_{p}\right)^{\exp }=1.293333(33) \mathrm{MeV}$, [2]. Including the $s$ quark then the flavour symmetry group becomes $S U(3)$ and the (pseudoscalar) mesons and baryons can be arranged in representations of this group. In Fig. 1 we show the lowest octet baryon and pseudoscalar states. States at the center, for example $\Lambda(u d s), \Sigma^{0}(u d s)$ have the
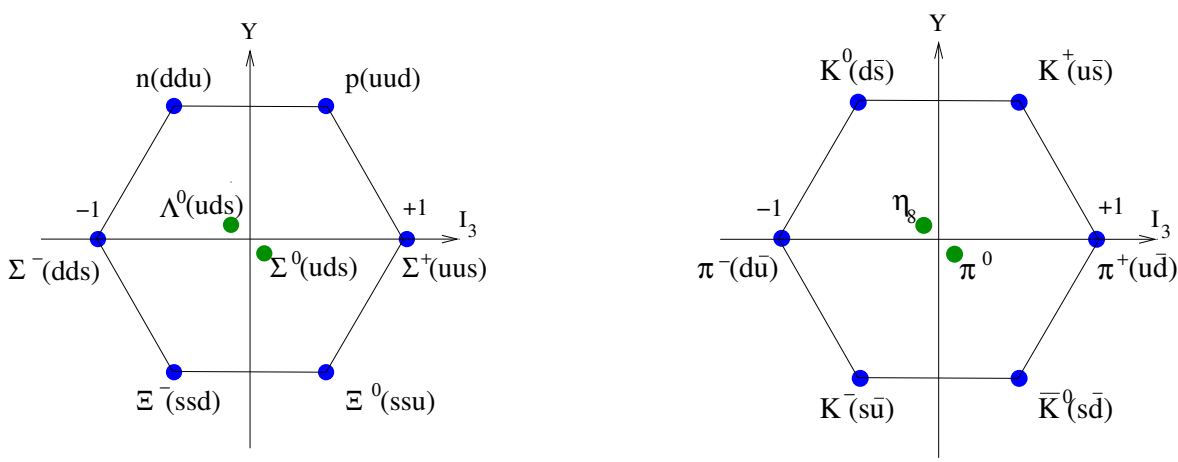

Figure 1: The (lowest) octet baryon states, left panel and the octet pseudoscalar states, right panel.

same quark content (quantum numbers) but different wavefunctions and can mix (if isospin is broken) so we shall only consider states on the 'outer' ring here. As well as $n-p$ mass splitting we now also have mass splittings involving the strange quark, $\left(M_{\Sigma^{-}}-M_{\Sigma^{+}}\right)^{\exp }=8.079(76) \mathrm{MeV}$ and $\left(M_{\Xi^{-}}-M_{\Xi^{0}}\right)^{\exp }=6.85(21) \mathrm{MeV}$. These are all small differences (compared to the masses of the states) and the experimental precision is way beyond what we achieve here, but nevertheless we can qualitatively and reasonably quantitatively describe these splittings, as briefly described in the next section. For more details see [1].

\section{Method}

The QCDSF-UKQCD strategy is to develop an $S U(3)$ flavour symmetry breaking expansion, [3], from the flavour symmetric point down to the physical point. For the baryons on the outer ring 
of the octet we have found up to NNLO

$$
\begin{aligned}
M^{2}(a a b)=M_{0}^{2} & +A_{1}\left(2 \delta \mu_{a}+\delta \mu_{b}\right)+A_{2}\left(\delta \mu_{b}-\delta \mu_{a}\right) \\
& +\frac{1}{6} B_{0}\left(\delta m_{u}^{2}+\delta m_{d}^{2}+\delta m_{s}^{2}\right) \\
& +B_{1}\left(2 \delta \mu_{a}^{2}+\delta \mu_{b}^{2}\right)+B_{2}\left(\delta \mu_{b}^{2}-\delta \mu_{a}^{2}\right)+B_{3}\left(\delta \mu_{b}-\delta \mu_{a}\right)^{2} \\
& +C_{0} \delta m_{u} \delta m_{d} \delta m_{s} \\
& +\left[C_{1}\left(2 \delta \mu_{a}+\delta \mu_{b}\right)+C_{2}\left(\delta \mu_{b}-\delta \mu_{a}\right)\right]\left(\delta m_{u}^{2}+\delta m_{d}^{2}+\delta m_{s}^{2}\right) \\
& +C_{3}\left(\delta \mu_{a}+\delta \mu_{b}\right)^{3}+C_{4}\left(\delta \mu_{a}+\delta \mu_{b}\right)^{2}\left(\delta \mu_{a}-\delta \mu_{b}\right) \\
& +C_{5}\left(\delta \mu_{a}+\delta \mu_{b}\right)\left(\delta \mu_{a}-\delta \mu_{b}\right)^{2}+C_{6}\left(\delta \mu_{a}-\delta \mu_{b}\right)^{3},
\end{aligned}
$$

while for the pseudoscalar meson octet,

$$
\begin{aligned}
M^{2}(a \bar{b})=M_{0 \pi}^{2} & +\alpha\left(\delta \mu_{a}+\delta \mu_{b}\right) \\
& +\beta_{0} \frac{1}{6}\left(\delta m_{u}^{2}+\delta m_{d}^{2}+\delta m_{s}^{2}\right)+\beta_{1}\left(\delta \mu_{a}^{2}+\delta \mu_{b}^{2}\right)+\beta_{2}\left(\delta \mu_{a}-\delta \mu_{b}\right)^{2} \\
& +\gamma_{0} \delta m_{u} \delta m_{d} \delta m_{s}+\gamma_{1}\left(\delta \mu_{a}+\delta \mu_{b}\right)\left(\delta m_{u}^{2}+\delta m_{d}^{2}+\delta m_{s}^{2}\right) \\
& +\gamma_{2}\left(\delta \mu_{a}+\delta \mu_{b}\right)^{3}+\gamma_{3}\left(\delta \mu_{a}+\delta \mu_{b}\right)\left(\delta \mu_{a}-\delta \mu_{b}\right)^{2}
\end{aligned}
$$

We have defined for the sea quarks $\delta m_{q}=m_{q}-\bar{m}$ with $\bar{m}=\frac{1}{3}\left(m_{u}+m_{d}+m_{s}\right)$, where $q \in\{a, b, \ldots\}$ so at the $S U$ (3) flavour symmetric point $\delta m_{q}=0$. From this definition this means that $\delta m_{s}=$ $-2 \delta m_{l}$. All the expansion coefficients are functions of $\bar{m}$ only. For the baryon or meson valence quarks we allow partially quenching, $\mathrm{PQ}$, and set $\delta \mu_{q}=\mu_{q}-\bar{m}$ (i. e. valence quark masses $\mu_{q} \neq$ sea quark masses $m_{q}$ ). Of course on the unitary line when the sea and valence quark masses are the same then $\delta \mu_{q} \rightarrow \delta m_{q}$. The quarks $q=a, b, \ldots$ are from $\{u, d, s\}$, so for example $M(u u d)=M_{p}$, $M(d d s)=M_{\Sigma^{-}}$. We shall also need pseudoscalar mass results and the corresponding $S U(3)$ flavour breaking expansion to determine the physical point: $\delta m_{d}^{*}, \delta m_{u}^{*}$ and $\delta m_{s}^{*}$ (where a ${ }^{*}$ denotes the physical point).

On the unitary line, singlet quantities have the property that the leading $O\left(\delta m_{q}\right)$ term vanishes. This allows a relatively simple definition of the scale, as practically we have shown, [3], that these quantities hardly vary in the interval from the flavour symmetric point down to the physical point. There are many possibilities for example for octet baryons (which are all stable under strong interactions), we may consider the 'centre of mass' of the octet

$$
X_{N}^{2}=\frac{1}{6}\left(M_{p}^{2}+M_{n}^{2}+M_{\Sigma^{+}}^{2}+M_{\Sigma^{-}}^{2}+M_{\Xi^{0}}^{2}+M_{\Xi^{-}}^{2}\right)=M_{0}^{2}+O\left(\delta m_{q}^{2}\right),
$$

and similarly for the octet of pseudoscalar mesons

$$
X_{\pi}^{2}=\frac{1}{6}\left(M_{K^{+}}^{2}+M_{K^{0}}^{2}+M_{\pi^{+}}^{2}+M_{\pi^{-}}^{2}+M_{\bar{K}^{0}}^{2}+M_{K^{-}}^{2}\right)=M_{0 \pi}^{2}+O\left(\delta m_{q}^{2}\right) .
$$

Using this we can form ratios $\tilde{M} \equiv M / X_{S}$ for $S=N, \pi$ with expansion coefficients $\tilde{A}_{i} \equiv A_{i} / M_{0}^{2}, \tilde{\alpha} \equiv$ $\alpha / M_{0 \pi}^{2}, \ldots$ for the $S U(3)$ flavour breaking expansions.

Note that as the coefficients of the $S U(3)$ flavour breaking expansions are just functions of $\bar{m}$ alone, so provided $\bar{m}$ remains constant, the coefficients can be determined by $n_{f}=2+1$ simulations, when $\delta m_{u}=\delta m_{d} \equiv \delta m_{l}$ rather than more expensive $n_{f}=1+1+1$ simulations. Also an 
additional advantage is that computationally cheaper PQ results can help to determine the coefficients.

Using $O(a)$-improved clover fermions, [4], at $\beta=5.50$ we have determined the appropriate point on the $S U$ (3) flavour symmetric line (for the path to the physical point) and then used this point for PQ determinations of heavier baryon and meson masses (so that $\bar{m}$ obviously remains constant). Fitting these masses (and also including unitary data at the same constant $\bar{m}$ ) then allows determinations of the expansion coefficients. In general these fits are functions of two quark masses $\delta \mu_{a}$ and $\delta \mu_{b}$. To avoid a 3-dimensional plot, as an illustration of these fits in Fig. 2 we show
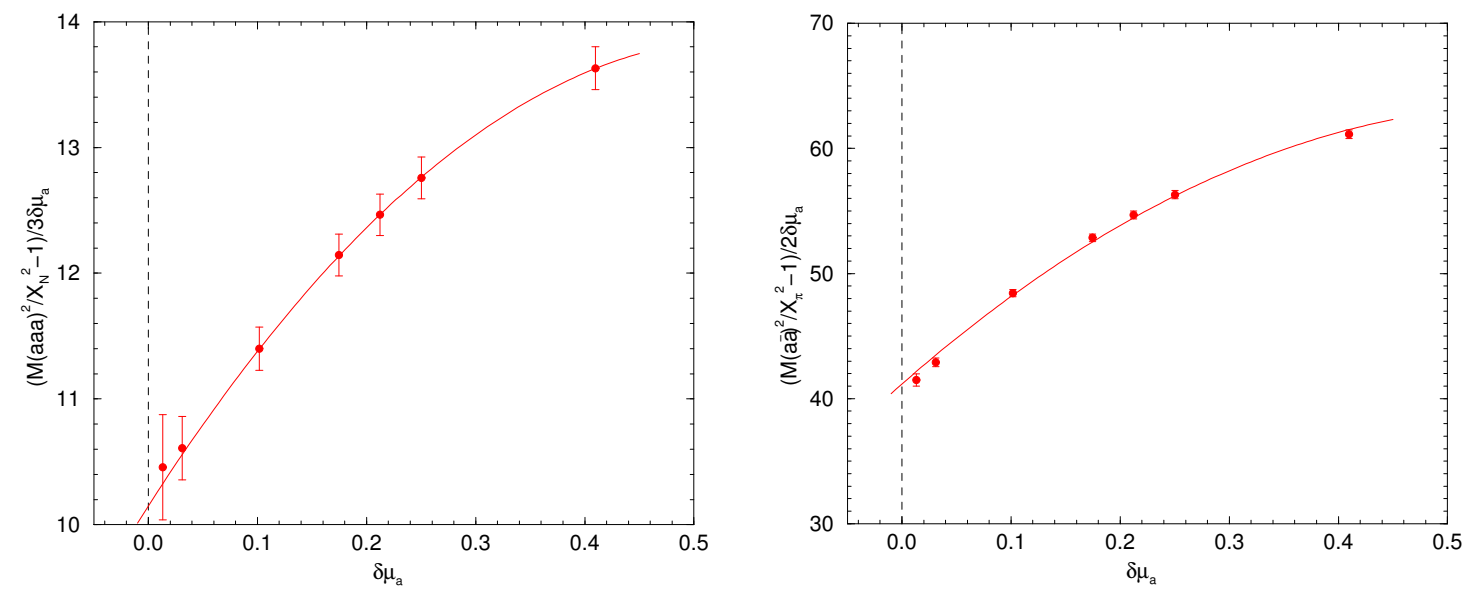

Figure 2: The mass degenerate results, octet baryon $\tilde{M}^{2}(a a a)$ left panel and octet pseudoscalar meson $\tilde{M}^{2}(a \bar{a})$ right panel (using $32^{3} \times 64$ sized lattices), both graphs versus the PQ quark mass $\delta \mu_{a}$. Also shown are the functions given in eq. (2.5).

$\left(\tilde{M}^{2}(a a a)-1\right) /\left(3 \delta \mu_{a}\right)$ (left panel) and $\left(\tilde{M}^{2}(a \bar{a})-1\right) /\left(2 \delta \mu_{a}\right)$ (right panel), together with the fit functions derived from eqs. (2.1) - (2.2) by taking completely degenerate quark masses

$$
\frac{\tilde{M}^{2}(a a a)-1}{3 \delta \mu_{a}}=\tilde{A}_{1}+\tilde{B}_{1} \delta \mu_{a}+\frac{8}{3} \tilde{C}_{3} \delta \mu_{a}^{2}, \quad \frac{\tilde{M}^{2}(a \bar{a})-1}{2 \delta \mu_{a}}=\tilde{\alpha}_{1}+\tilde{\beta}_{1} \delta \mu_{a}+4 \tilde{\gamma}_{2} \delta \mu_{a}^{2} .
$$

As another example, we also consider the unitary results (i.e. $\delta \mu_{q} \rightarrow \delta m_{q}$ ) from the $S U(3)$ flavour symmetric point down to the physical point. In Fig. 3 we show the baryon and pseudoscalar octet 'fan' plots, where $M_{N} \equiv M(l l l), M_{\Sigma} \equiv M(l l s), M_{\Xi} \equiv M(l s s), M_{N_{s}} \equiv M(s s s)$ and $M_{\pi} \equiv M(l \bar{l})$, $M_{K} \equiv M(l \bar{s}), M_{\eta_{s}} \equiv M(s \bar{s})$. The states $M_{N_{s}}, M_{\eta_{s}}$ are either not in the octet or are a PQ state and are not physical, but nevertheless can be used to help determine the expansion coefficients. The vertical lines are the $n_{f}=2+1$ pure QCD physical point, with the opaque circles being the determined pure QCD hadron mass ratios for $2+1$ quark flavours. For comparison, the stars represent the average of the squared masses of the appropriate particle on the outer ring of the baryon octet, Fig. 1, i.e. $M_{N}^{* 2}(l l l)=\left(M_{n}^{\exp 2}(d d u)+M_{p}^{\exp 2}(u u d)\right) / 2, M_{\Sigma}^{* 2}(l l s)=\left(M_{\Sigma^{-}}^{\exp 2}(d d s)+M_{\Sigma^{+}}^{\exp 2}(u u s)\right) / 2$, $M_{\Xi}^{* 2}(s s l)=\left(M_{\Xi^{-}}^{\exp 2}(s s d)+M_{\Xi^{0}}^{\exp 2}(s s u)\right) / 2$. One immediate observation of Fig. 3 is that there is hardly any curvature in the data and that the NLO (i.e. quadratic terms) are sufficient.

Finally note that the $x$-scales used in Fig. 2 and Fig. 3 are very different $\left|\delta m_{l}\right| \sim 0.01 \ll \delta \mu_{a} \sim$ 0.5 . (Indeed $\delta \mu_{a} \sim 0.5$ is roughly at the charm quark mass.) The ability to use a large range for the PQ fits enables a much better determination of the fit coefficients (in particular the NLO terms, which are poorly determined in the narrow range $\left|\delta m_{l}\right| \sim 0.01$ ). 

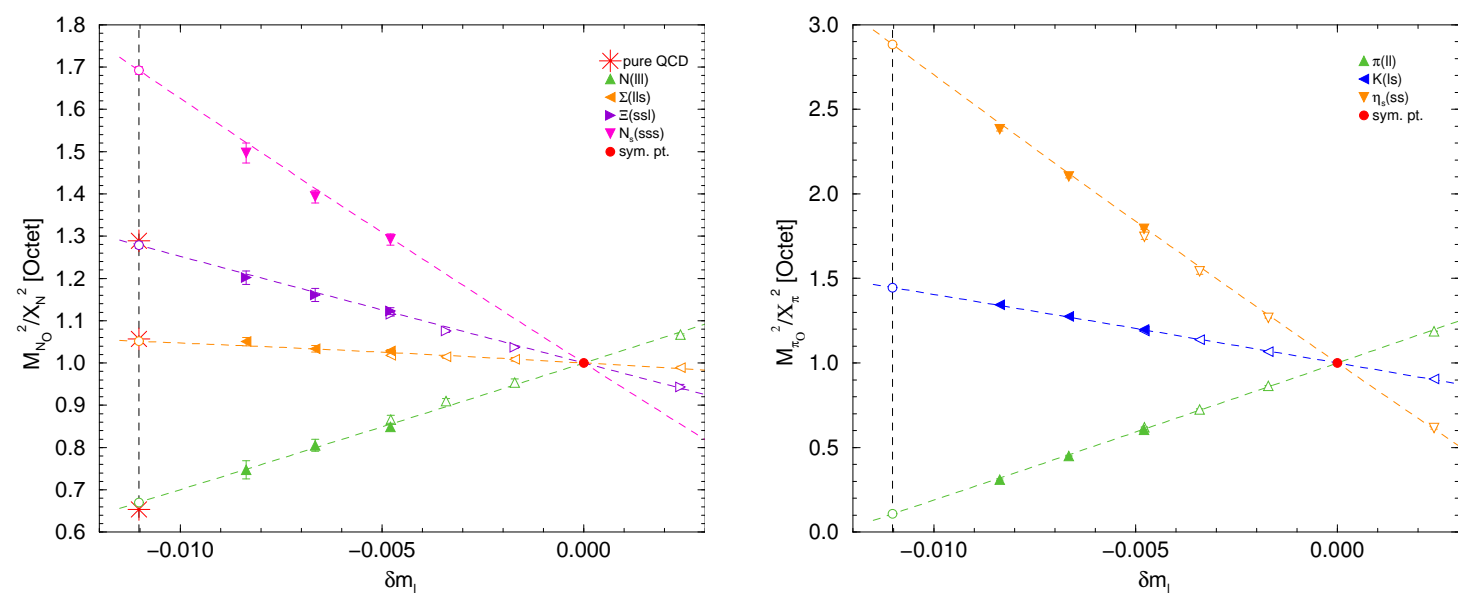

Figure 3: The baryon octet 'fan' plot, $\tilde{M}_{N_{O}}^{2} \equiv M_{N_{O}}^{2} / X_{N}^{2}\left(N_{O}=N, \Sigma, \Xi, N_{s}\right)$ left panel and the pseudoscalar meson octet 'fan' plot $\tilde{M}_{\pi_{O}}^{2} \equiv M_{\pi_{O}}^{2} / X_{\pi}^{2}\left(\pi_{O}=\pi, K, \eta_{s}\right)$ right panel, both graphs versus $\delta m_{l}$. The filled symbols represent mass values from $32^{3} \times 64$ sized lattices while the opaque symbols are from $24^{3} \times 48$ sized lattices (and not used in the fits here). The common symmetric point is the filled circle. The stars (on the vertical line) are the estimated $n_{f}=2+1$ 'pure' QCD physical points. The fits are eqs. (2.1) $-(2.2)$.

Of course, we are interested in mass differences here. So for the LO and NLO terms in eq. (2.1) we have along the unitary line

$$
\begin{aligned}
\tilde{M}_{n}-\tilde{M}_{p} & =\tilde{M}(d d u)-\tilde{M}(u u d) \\
& =\left(\delta m_{d}-\delta m_{u}\right)\left[\tilde{A}_{1}^{\prime}-2 \tilde{A}_{2}^{\prime}+\left(\tilde{B}_{1}^{\prime}-2 \tilde{B}_{2}^{\prime}\right)\left(\delta m_{d}+\delta m_{u}\right)\right], \\
\tilde{M}_{\Sigma^{-}}-\tilde{M}_{\Sigma^{+}} & =\tilde{M}(d d s)-\tilde{M}(u u s) \\
& =\left(\delta m_{d}-\delta m_{u}\right)\left[2 \tilde{A}_{1}^{\prime}-\tilde{A}_{2}^{\prime}+\left(2 \tilde{B}_{1}^{\prime}-\tilde{B}_{2}^{\prime}+3 \tilde{B}_{3}^{\prime}\right)\left(\delta m_{d}+\delta m_{u}\right)\right], \\
\tilde{M}_{\Xi^{-}}-\tilde{M}_{\Xi^{0}} & =\tilde{M}(s s d)-\tilde{M}(s s u) \\
& =\left(\delta m_{d}-\delta m_{u}\right)\left[\tilde{A}_{1}^{\prime}+\tilde{A}_{2}^{\prime}+\left(\tilde{B}_{1}^{\prime}+\tilde{B}_{2}^{\prime}+3 \tilde{B}_{3}^{\prime}\right)\left(\delta m_{d}+\delta m_{u}\right)\right],
\end{aligned}
$$

where the prime coefficients are simply related to the unprimed ones, [1]. Similarly we may invert the meson pseudoscalar expansion, eq. (2.2), to give

$$
\begin{aligned}
& \delta m_{d}-\delta m_{u}=\frac{\tilde{M}_{K^{0}}^{2}-\tilde{M}_{K^{+}}^{2}}{\tilde{\alpha}}\left(1+\frac{2\left(\tilde{\beta}_{1}+3 \tilde{\beta}_{2}\right)}{3 \tilde{\alpha}^{2}}\left(\frac{1}{2}\left(\tilde{M}_{K^{0}}^{2}+\tilde{M}_{K^{+}}^{2}\right)-\tilde{M}_{\pi^{+}}^{2}\right)\right), \\
& \delta m_{d}+\delta m_{u}=-\frac{2}{3 \tilde{\alpha}}\left(\frac{1}{2}\left(\tilde{M}_{K^{0}}^{2}+\tilde{M}_{K^{+}}^{2}\right)-\tilde{M}_{\pi^{+}}^{2}\right),
\end{aligned}
$$

and then substitute in the baryon expansion eq. (2.6) to give the 'pure' QCD result.

\section{Results}

Performing this substitution gives the numerical results, [1],

$$
\begin{aligned}
\tilde{M}_{n}-\tilde{M}_{p} & =0.0789(41)(34)\left(\tilde{M}_{K^{0}}^{2}-\tilde{M}_{K^{+}}^{2}\right)\left[1+0.0817(92)\left(\frac{1}{2}\left(\tilde{M}_{K^{0}}^{2}+\tilde{M}_{K^{+}}^{2}\right)-\tilde{M}_{\pi^{+}}^{2}\right)\right], \\
\tilde{M}_{\Sigma^{-}}-\tilde{M}_{\Sigma^{+}} & =0.2243(35)(92)\left(\tilde{M}_{K^{0}}^{2}-\tilde{M}_{K^{+}}^{2}\right)\left[1+0.0077(30)\left(\frac{1}{2}\left(\tilde{M}_{K^{0}}^{2}+\tilde{M}_{K^{+}}^{2}\right)-\tilde{M}_{\pi^{+}}^{2}\right)\right], \\
\tilde{M}_{\Xi^{-}}-\tilde{M}_{\Xi^{0}} & =0.1455(24)(59)\left(\tilde{M}_{K^{0}}^{2}-\tilde{M}_{K^{+}}^{2}\right)\left[1-0.0324(50)\left(\frac{1}{2}\left(\tilde{M}_{K^{0}}^{2}+\tilde{M}_{K^{+}}^{2}\right)-\tilde{M}_{\pi^{+}}^{2}\right)\right],(3.1
\end{aligned}
$$


where $\tilde{M}=M / X_{S}, S=N, \pi$. We see that the NLO corrections are small from $+10 \% \sim-5 \%$ indicating that the $S U(3)$ flavour symmetry breaking expansion appears to be a highly convergent series. (In eq. (3.1) the first error is statistical, the other is the total systematic error.)

Note that eq. (3.1) is a 'pure' QCD result, and is the main result of this talk. We must now discuss what are the 'pure' QCD values of $M_{K^{0}}^{2}, M_{K^{+}}^{2}, M_{\pi^{+}}^{2}$. We know that EM effects are comparable to effects due to $u-d$ quark mass differences. Dashen's theorem states that EM effects for charged mesons $K^{+}, \pi^{+}$are the same and for neutral mesons $\pi^{0}, K^{0}$ vanish, Thus we can write $M_{\pi^{+}}^{\exp 2}=M_{\pi^{+}}^{* 2}+\mu_{\gamma}, M_{\pi^{0}}^{\exp 2}=M_{\pi^{0}}^{* 2} \approx M_{\pi^{+}}^{* 2}, M_{K^{+}}^{\exp 2}=M_{K^{+}}^{* 2}+\mu_{\gamma}$ and $M_{K^{0}}^{\exp 2}=M_{K^{0}}^{* 2}$ where a ${ }^{*}$ denotes the 'pure' QCD 'physical' value, or $M_{K^{0}}^{* 2}-M_{K^{+}}^{* 2}=\left(M_{K^{0}}^{2}-M_{K^{+}}^{2}\right)^{\exp }+\left(1+\varepsilon_{\gamma}\right)\left(M_{\pi^{+}}^{2}-M_{\pi^{0}}^{2}\right)^{\exp }$, where violations to Dashen's theorem $\left(\varepsilon_{\gamma}=0\right)$ are given by a non-zero $\varepsilon_{\gamma}$. We shall regard $\varepsilon_{\gamma}$ here as a possible further systematic error, a typical value for it being $\varepsilon_{\gamma}=0.7$, [5], giving about a $17 \%$ additional systematic error.

Let us first investigate QED effects. From eq. (3.1), together with the numerical pseudoscalar meson masses from the previous paragraph we can determine the 'pure' QCD values. Then as we know the experimental values, [2] (as also given on page 2) then from eq. (1.1) we can determine the QED contribution to the mass splittings. This is shown in the left panel of Fig. 4. Thus this
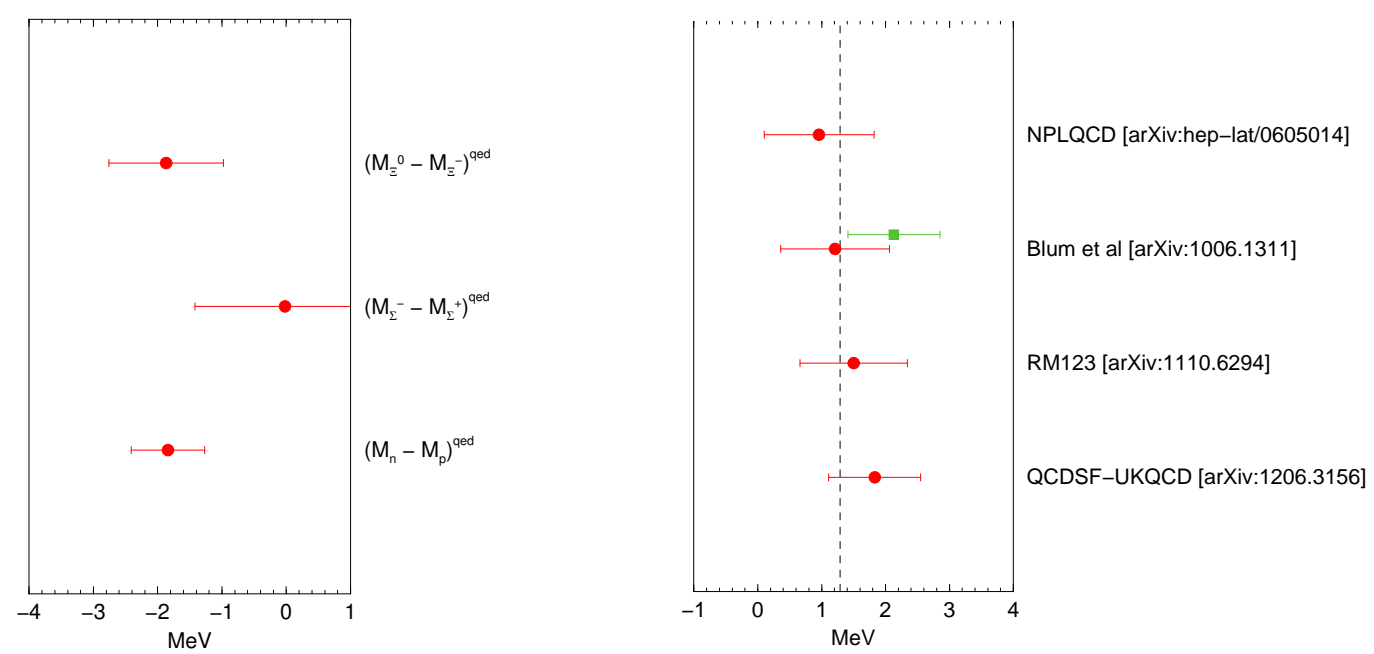

Figure 4: Left panel: QED contribution to the baryon octet mass splittings, filled circles. Right panel: comparison of the $n-p$ mass difference of the present result (QCDSF-UKQCD or bottom number) with NPLQCD, Blum et al., and RM123 [6, 7, 8] respectively (top to bottom). The filled circles use the QED determination of [9], while the filled square includes the full determination from Blum et al. The vertical dashed line is the experimental result.

indicates that EM effects have the pattern

$$
n(d d u)-p(u u d) \approx \Xi^{0}(s s d)-\Xi^{-}(s s u)<0, \quad \Sigma^{-}(d d s)-\Sigma^{+}(u u s) \approx 0 .
$$

Alternatively [9] gives a determination of electromagnetic effects of $n-p$ of $-1.30(47) \mathrm{MeV}$ (to be compared with $-1.84(57) \mathrm{MeV}$ here). In the right panel of Fig. 4 we compare our $n-p$ mass difference including this determination of the QED contribution, $\left(M_{n}-M_{p}\right)^{*+Q E D}$, bottom result with the results of $[6,7,8]$ (top to bottom). The filled square includes the full determination 
from that reference. Despite the fact that QED effects are treated slightly differently in each work good agreement amongst the various determinations and with the experimental result is found.

\section{Conclusions}

We have introduced a method here to determine 'pure' QCD isospin effects in

$$
n-p, \quad \Sigma^{-}-\Sigma^{+}, \quad \Xi^{-}-\Xi^{0},
$$

due to differences in $u-d$ quark masses. This method involves developing a $S U$ (3) flavour symmetry breaking expansion keeping the average quark mass $\bar{m}$ constant. Advantages include the ability to use $2+1$ simulations, i.e. $m_{u}=m_{d}=m_{l}$ and use of computationally cheap PQ results. This expansion appears to be highly convergent, giving encouraging first results. Clearly the largest errors are due to unknown QED effects. For more details and numerical results see [1].

\section{Acknowledgements}

The numerical configuration generation was performed using the BQCD lattice QCD program, [10], on the IBM BlueGeneL at EPCC (Edinburgh, UK), the BlueGeneL and P at NIC (Jülich, Germany), the SGI ICE 8200 at HLRN (Berlin-Hannover, Germany) and the JSCC (Moscow, Russia). The BlueGene codes were optimised using Bagel. The Chroma software library, was used in the data analysis. This investigation has been supported partly by the DFG under contract SFB/TR 55 (Hadron Physics from Lattice QCD) and by the EU grants 283286 (Hadron Physics3), 227431 (Hadron Physics2) and 238353 (ITN STRONGnet). JN was partially supported by EU grant 228398 (HPC-EUROPA2). JMZ is supported by the Australian Research Council grant FT100100005. We thank all funding agencies.

\section{References}

[1] R. Horsley et al., [QCDSF-UKQCD Collaboration], arXiv:1206.3156 [hep-lat ] .

[2] K. Nakamura et al., (Particle Data Group), J. Phys. G 37 (2010) 075021.

[3] W. Bietenholz et al., [QCDSF-UKQCD Collaboration], Phys. Rev. D84 054509 (2011), [arXiv:1102.5300 [hep-lat]].

[4] N. Cundy et al., [QCDSF-UKQCD Collaboration], Phys. Rev. D79 (2009) 094507, [arXiv:0901.3302 [hep-lat]].

[5] G. Colangelo et al., [FLAG], Eur. Phys. J. C71 (2011) 1695, [arXiv:1011. 4408 [hep-lat ] ].

[6] S. R. Beane et al., Nucl. Phys. B768 (2007) 38, [arXiv: hep-lat/0605014].

[7] T. Blum et al., Phys. Rev. D82 (2010) 094508, [arXiv:1006.1311 [hep-lat] ].

[8] G. M. de Divitiis et al., [RM123 Collaboration], JHEP 1204 (2012) 124, [arXiv:1110.6294[hep-lat]].

[9] A. Walker-Loud et al., Phys. Rev. Lett. 108 (2012) 232301, [arXiv:1203. 0254 [nucl-th]].

[10] Y. Nakamura et al., PoS(Lattice 2010) 040 (2010), arXiv: 1011.0199 [ hep-lat ] . 